EPSC Abstracts

\title{
The UK Fireball Alliance (UKFAlI); combining and integrating the diversity of UK camera networks to aim to recover the first UK meteorite fall for $\mathbf{3 0}$ years
}

Luke Daly 1,2,3, Sarah McMullan ${ }^{4,5}$, Jim Rowe ${ }^{6}$, Gareth S. Collins ${ }^{4,5}$, Martin Suttle ${ }^{4,7}$, Queenie H.S. Chan $^{4,8}$, John S. Young ${ }^{4,9}$, Clive Shaw ${ }^{4,10}$, Adrian G. Mardon ${ }^{4,11}$, Mike Alexander ${ }^{4,12}$, Jonathan Tate $^{4,13}$, The Desert Fireball Network Team ${ }^{2}$, Peter Campbell-Burns ${ }^{14}$, Richard Kacerek ${ }^{14}$, Ashley King $^{15}$, Katherine Joy ${ }^{16}$, Apostolos Christou ${ }^{17}$, Jana Horák ${ }^{18}$, and Jamie Shepherd

${ }^{1}$ University of Glasgow, School of Geographical and Earth Sciences, United Kingdom of Great Britain and Northern Ireland (luke.daly@glasgow.ac.uk)

${ }^{2}$ Space Science and Technology Centre, School of Earth and Planetary Sciences, Curtin University, GPO Box U1987, Perth, WA, 6845, Australia.

${ }^{3}$ Australian Centre for Microscopy and Microanalysis, The University of Sydney, NSW, 2006, Australia.

${ }^{4}$ UK Fireball Network.

${ }^{5}$ Impact and Astromaterials Research Centre, Department of Earth Science and Engineering, Imperial College London, SW7 2AZ, UK.

${ }^{6}$ SCAMP fireball network, UK.

${ }^{7}$ Natural History Museum, London, SW7 5BD, UK.

${ }^{8}$ Department of Earth Sciences, Royal Holloway University, Egham Hill, Egham TW20 0EX, UK.

${ }^{9}$ University of Cambridge, Mullard Radio Astronomy Observatory, Cambridge Road, Harlton, Cambridgeshire, CB23 1EX, UK.

${ }^{10}$ Cavendish Laboratory, University of Cambridge, Cambridge CB3 OHE, UK.

${ }^{11}$ Newby Hall and Gardens, Ripon, Yorkshire, HG4 5AE, UK.

${ }^{12}$ Galloway Astronomy Centre, Glasserton, Nr Whithorn, DG8 8NE, UK.

${ }^{13}$ The Spaceguard Centre, Llanshay Lane, Knighton, LD7 1LW, UK.

${ }^{14}$ UK Meteor Observation Network, UK.

${ }^{15}$ School of Physical Sciences, The Open University, Walton Hall, Milton Keynes, MK7 6AA, UK.

${ }^{16}$ School of Earth \& Environmental Sciences, The University of Manchester, Oxford Rd, Manchester M13 9PL, UK.

${ }^{17}$ Armagh Observatory and Planetarium, College Hill, Armagh BT61 9DB, UK.

${ }^{18}$ Mineralogy \& Petrology, National Museum Wales, Cathays Park, Cardiff, CF10 3NP.

\section{Main text}

The UK has a long history of meteorite falls (where the meteorite fireball is witnessed, and the stone recovered, dating back to 1623 (MetBull, 2020). But the last meteorite fall in the UK was nearly 30 years ago when the Glatton stone, an L6 ordinary chondrite was recovered in 1991 (Hutchinson et al., 1991). Meteorite falls are important samples as they are usually recovered within days of the fireball event. As such, they have not experienced the deleterious effects terrestrial weathering that can change their extraterrestrial mineralogy, chemistry and isotopic composition (Bland et al., 2006). In exceptional circumstances rapidly recovered falls may have avoided rainfall so that soluble extraterrestrial minerals such as salts may be preserved (Chan et al., 2018). Therefore, meteorite falls represent much more pristine extraterrestrial material than their find counterparts within the same group, and consequently, characterisation of their texture and chemical signatures provides a clearer window into solar system processes. However, even falls are limited in their interpretive 
power as the geological context of the stone (i.e. where in the solar system it originated from) is unknown.

To derive this contextual information requires the imaging of the fireball event from multiple geographical positions (Devillepoix et al., 2020). This data provides two vital pieces of information; the initial orbit of the meteoroid can be calculated and the final fall position can be predicted with increased accuracy (Devillepoix et al., 2020). As such, dedicated fireball camera networks (Bowden, 2006) are entering 'a golden age' with improvements in hardware and software capabilities as well as a reduction in production costs (Spurný et al., 2014). Continent-scale observatories have been established (Howie et al., 2017) and global networks are under construction (Devillepoix et al., 2020). In addition, these same developments have enabled the amateur astronomy community to construct their own networks either as groups or individuals with functional data pipelines and observations that can rival funded networks. Consequently, the number of recovered meteorite falls with orbits globally has grown rapidly over recent years (Borovička et al., 2015).
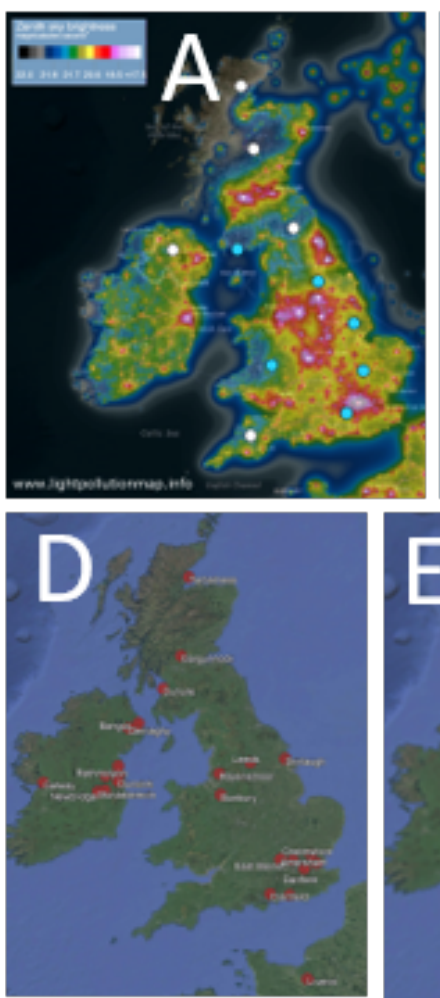
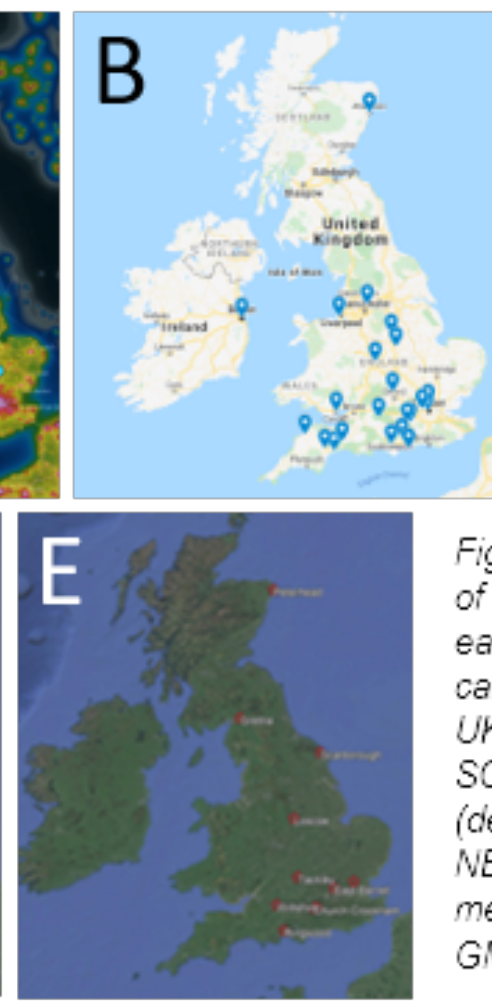

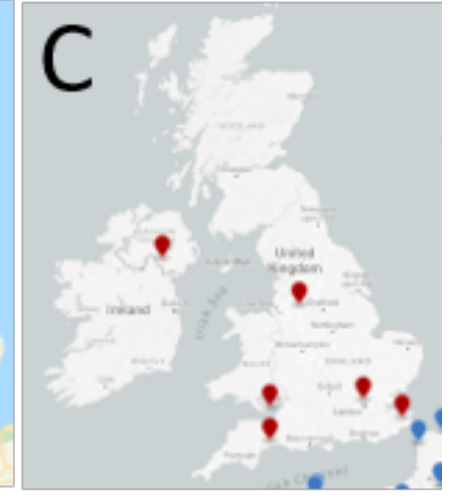

Figure 1. The distribution of observatories from each of the UK's fireball camera networks A) UKFN. B) UKMON. C) SCAMP D) NEMATODE (derived from NEMATODE observed meteor data set 2018. E) GMN.

The UK is a hotbed for such activity but does not have a recent recovered meteorite fall...yet. There are currently two active academically funded networks in the UK: the UK Fireball Network (UKFN: part of the Global Fireball Observatory built on the hardware and software developed by the Desert Fireball Network in Australia), and the System for Capture of Asteroid and Meteorite Paths (SCAMP; the UK arm of the French-led Fireball Recovery and InterPlanetary Observation Network (FRIPON)) (Figure 1, 2). In addition, there are two major amateur networks the UK Meteor Observation Network (UKMON), and NEMETODE, as well as an emerging presence of the Raspberry-Pi based Global Meteor Network and countless individual citizen scientists also imaging the UK night skies (Figure 1, 2).

However, environmental factors in the UK such as light pollution and regular low-lying cloud cover means that a single fireball may not be captured multiple times by one network, preventing them from calculating an orbit and accurate fall position. As such, these networks, together with UK planetary scientists and National museums, have formed a collaborative data-sharing initiative called the UK Fireball Alliance (UKFAll) in order to maximise the chances of capturing a meteorite- 

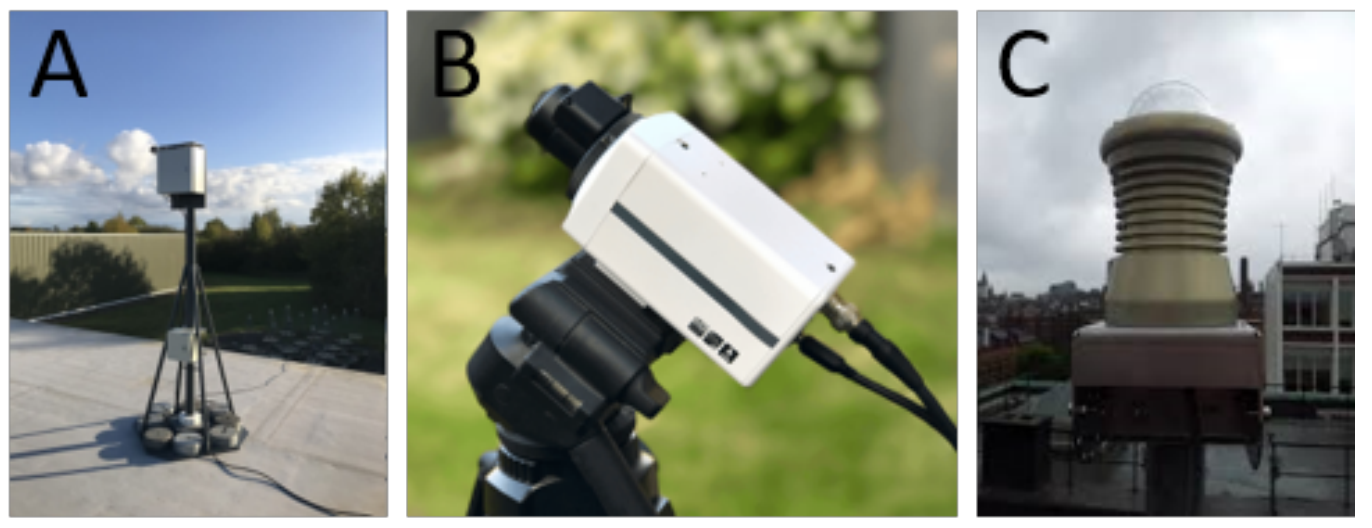

Figure 2. The diversity of observotory infrostructure present in the UK's fireboll networks. A) UKFN all sky digital camero. B) UKMON narrow angle CCT video camero. C) FRIPON all sky video comera.

Since the initiation of UKFAll in late 2018 many joint fireball observations have been made between UK networks including a fireball on the $16^{\text {th }}$ February 2020 that likely dropped a few 10 s of grams of extraterrestrial material into the North Sea (Figure 3).

However, the diversity of hardware, software and data processing pipelines for capturing fireball events vary between camera networks. This hinders the speed of detecting and triangulating a meteorite-dropping fireball event as each fireball requires a bespoke solution to translate the data into the other networks' format. A consistent method for rapidly transferring and converting the diversity of outputs produced by each network into a standard format that can be read and utilised by each network is required and is critical to facilitate a rapid UK response to fireball events and associated recovery effort.

Here we describe the first iteration of a new code that will enable rapid conversion of data outputs from both video and still image camera networks. The code provides an effective bridging solution, while the ultimate aim is to agree and implement a globally accepted standardised format for fireball observations that can be readily transferred and utilised between camera networks to facilitate meteorite fall recovery.

We also describe the logistical issues encountered by UKFAll and the solutions being implemented, including: recruitment of citizen scientists as searchers; conduct and liability issues; and best practice for collection. 

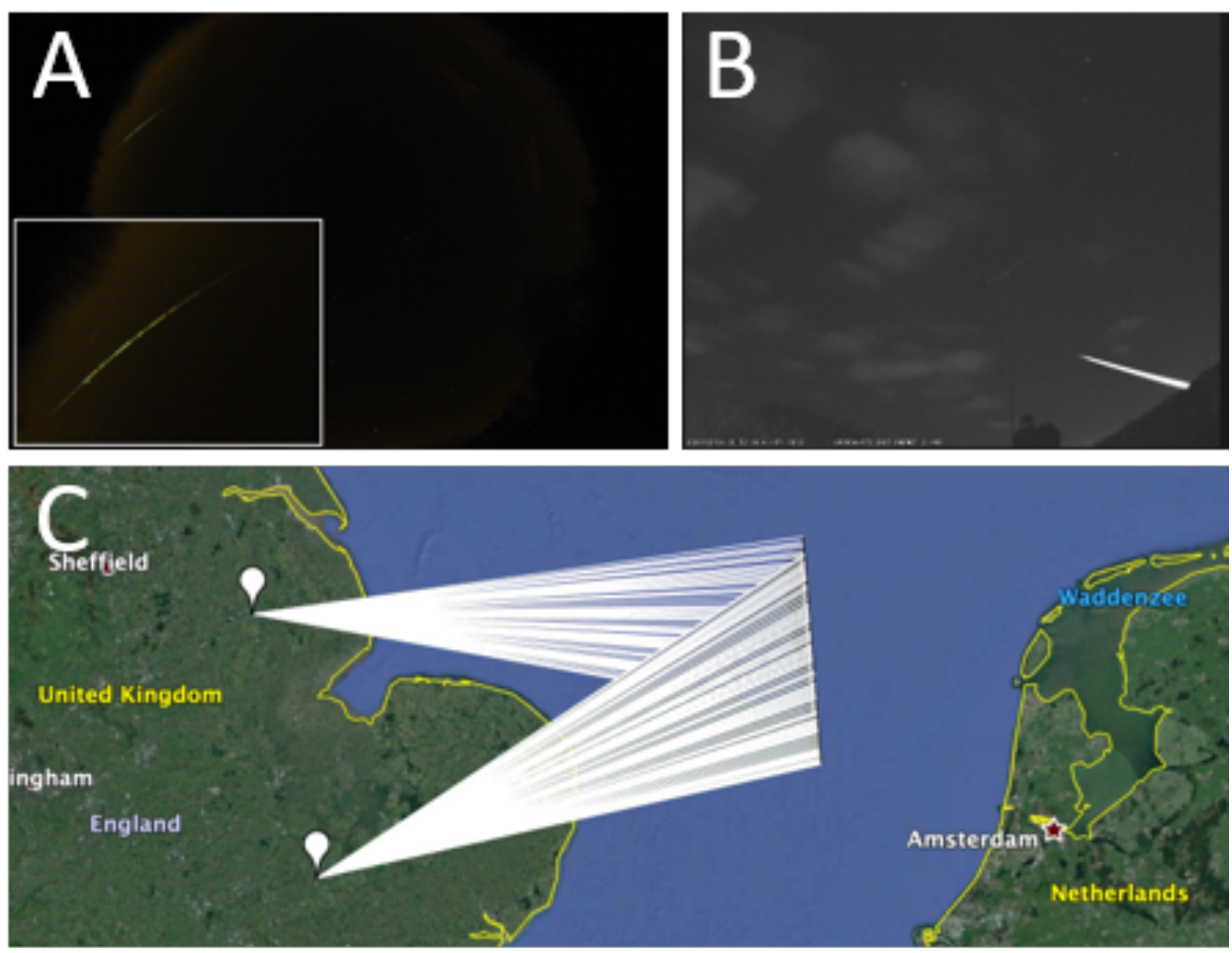

Figure 3. The meteorite dropping fireball imaged on the $15^{\text {th }}$ February 2020 by all camero networks. A) The all sky image and thumbnoil of the fireball as coptured by the UKFN oll sky camero at Mullard Observotory Combrioge. B) The some Fireball event (16 Feb) as seen from the UKMON's East Bornet camero. C) Triangulation of the bright flight of the fireboll from two UKFN observatories.

\section{References}

Bland, P.A., et al., (2006). Weathering of chondritic meteorites. Meteorites and the early solar system II, 1, 853-867.

Borovička J., et al., (2015). Small near $\square$ Earth asteroids as a source of meteorites. In Asteroids IV, edited by Michel P., DeMeo F.E., and Bottke W.F. Tucson, Arizona: University of Arizona Press. pp. 257-280.

Bowden, A.J. 2006. "Meteorite provenance and the asteroid connection". In The history of meteoritics and key meteorite collections; fireballs, falls and finds, Edited by: G.J.H., McCall, A.J., Bowden and R.J., Howarth. Vol. 256, 379-403. Geological Society, London, Special Publications.

Chan, Q.H., et al., (2018). Organic matter in extraterrestrial water-bearing salt crystals. Science advances, 4(1), eaao3521.

Devillepoix, H.A., et al., (2018). The dingle dell meteorite: a halloween treat from the main belt. Meteoritics \& Planetary Science, 53(10), 2212-2227.

Devillepoix, H.A.R., et al., (2020). A Global Fireball Observatory. arXiv preprint arXiv:2004.01069.

Howie, R.M., et al., (2017). How to build a continental scale fireball camera network. Experimental Astronomy, 43(3), 237-266.

Hutchison, R., et al., (1991). The L6 chondrite fall at Glatton, England, 1991 May 5. Meteoritics, 26, 349. 
MetBull (2020), The meteoritical bulletin database. URL:

https://www.lpi.usra.edu/meteor/metbull.php

Spurný P., et al., (2014). Reanalysis of the Benešov bolide and recovery of polymict breccia meteorites-Old mystery solved after 20 years. Astronomy \& Astrophysics 570:A39. 